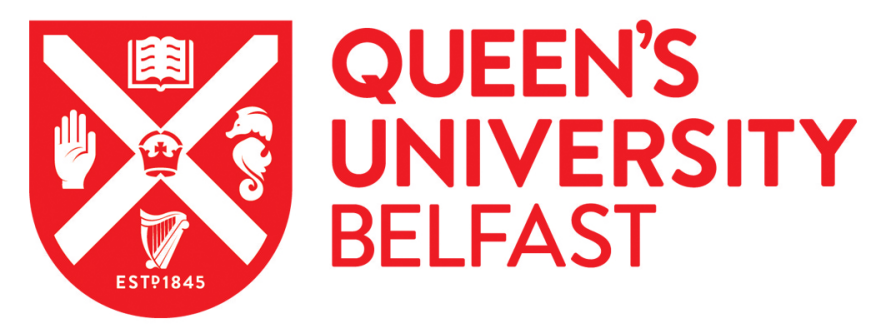

\title{
The Impact of a Policy-Based Multicomponent Nutrition Pilot Intervention on Young Adult Employee's Diet and Health Outcomes
}

Schliemann, D., McKinley, M., \& Woodside, J. V. (2018). The Impact of a Policy-Based Multicomponent Nutrition Pilot Intervention on Young Adult Employee's Diet and Health Outcomes. AMERICAN JOURNAL OF HEALTH PROMOTION. https://doi.org/10.1177/0890117118784447

Published in:

AMERICAN JOURNAL OF HEALTH PROMOTION

Document Version:

Peer reviewed version

Queen's University Belfast - Research Portal:

Link to publication record in Queen's University Belfast Research Portal

Publisher rights

( 2018 The Authors. This work is made available online in accordance with the publisher's policies. Please refer to any applicable terms of use of the publisher.

\section{General rights}

Copyright for the publications made accessible via the Queen's University Belfast Research Portal is retained by the author(s) and / or other copyright owners and it is a condition of accessing these publications that users recognise and abide by the legal requirements associated with these rights.

Take down policy

The Research Portal is Queen's institutional repository that provides access to Queen's research output. Every effort has been made to ensure that content in the Research Portal does not infringe any person's rights, or applicable UK laws. If you discover content in the Research Portal that you believe breaches copyright or violates any law, please contact openaccess@qub.ac.uk. 


\section{Purpose}

Workplace diet interventions have been found effective in improving employees' diet, particularly fruit and vegetable (FV) consumption ${ }^{1,2}$. Interventions including multiple components, such as education, individual and environmental components have been suggested to be most effective ${ }^{1,3}$. However, often such interventions implement changes in medium or large workplaces with food-serving canteens, and are not always applicable to small- and medium-size enterprises without canteens. There is a need to explore interventions for these businesses that nudge employees to eat healthier. Furthermore, previous workplacebased research mainly looked at middle-aged adults and little research has been carried out in young working adults. Encouraging healthy eating in young adults, however, may lay the foundations for a healthy ageing population. The term 'young adults' often refers to adults aged 18-25 or 18-35 years. The majority of diet interventions in young adults have looked at university students ${ }^{4,5}$ and may not be applicable for young working adults. Commonly reported diet behaviors amongst students include sugar-sweetened beverage consumption ${ }^{6}$, meal-skipping, snacking, large portion sizes and low FV consumption ${ }^{7}$. A systematic review (SR) found that young adults consume more foods out of home compared to older age groups, which was associated with higher total energy (TE) intake, higher energy intake from fat and lower micronutrient consumption ${ }^{8}$. To improve student's diets, educational diet interventions have been suggested effective ${ }^{9}$, though evidence for intervention effectiveness in young working adults is lacking. This study aimed to (I) explore the effectiveness of a workplace-based multi-component nutrition intervention in a medium-sized workplace, that does not have a canteen, on diet- and health-related outcomes of young adult employees and (II) conduct a process evaluation that involved employees and managers from the company in identifying successful components of, as well as barriers to, the intervention. The primary endpoint of this study was to examine the effect of the intervention on the diet of employees during the working week (ON duty) and on weekends (OFF duty). Secondary outcomes were (a) health measures, i.e. body weight, body mass index (BMI), midway waist circumference (WC) and blood pressure; 
(b) biomarkers, i.e. urinary Vitamin C; (c) factors that may influence dietary behavior change, i.e. diet knowledge; (d) work-related factors, i.e. job satisfaction, retention and sickness absenteeism.

\section{Methods}

\section{Design}

This was a single-armed pilot study that tested a dietary intervention with a mixture of policy, environmental, educational and individual behavior change components over a six-month period (Figure 1). The intervention was designed following the MRC guidelines ${ }^{10}$. Ethical approval for this study was granted by the Ethics Committee of the School of Medicine, Dentistry and Biomedical Sciences, Queen's University Belfast (Ref. 15.12).

\section{Sample}

The study population was recruited from an insurance company in Belfast, Northern Ireland who employed 83 staff. Employees were introduced to the study through an introductory talk by the researchers which was accompanied by a healthy breakfast. All employees who agreed to participate, met the inclusion criteria and signed the consent form were included. Since this was a pilot study, a power calculation was not carried out.

Inclusion criteria: (a) Employees working at the company throughout all assessments and the full intervention period.

Exclusion criteria: (a) Women who were pregnant or breastfeeding; (b) Employees who were on a weightreducing or weight-gaining diet 


\section{Measures}

Participants attended two appointments at baseline and at follow-up, during working hours. Figure 2 demonstrates the measurements taken at each appointment.

\section{Health assessments}

Weight and height measures were used to calculated BMI $\left(\mathrm{kg} / \mathrm{m}^{2}\right)$. The average distance between the last rib and the hipbone was taken and from there the midway WC was measured. Systolic (SBP) and diastolic blood pressure (DBP) readings were obtained using an OMRON M5-1 digital blood pressure monitor. All readings were taken three times to increase accuracy and the average was calculated from the second and third reading.

\section{Dietary assessments}

At baseline and follow-up, two 24-h diet recalls were conducted. One recall was taken on a Monday and one on a different weekday to account for the differences between weekend (OFF duty) and weekday (ON duty) eating habits. The 24-h diet recall was an adapted version of the validated UK standard 24-hour diet recall as used previously for workplace diet interventions ${ }^{11}$. Each recall took approximately 20 minutes. Further questions to examine diet intake over one week were added to the questionnaire, e.g. 'In a typical week, on how many days do you eat fruit?' and 'How many servings of fruit do you eat on one of those days?' 


\section{Work-related measures}

Sickness absenteeism and staff retention was assessed by the company. Job satisfaction was assessed as part of the study questionnaire.

\section{Study Questionnaires}

Participants completed a questionnaire at each time point, which contained a number of validated questionnaires and was a modified version of a questionnaire used previously ${ }^{11}$, i.e. the Health, Food and Lifestyle questionnaire collects information on demographic characteristics, working habits and general health as well as on diet, physical activity (International Physical Activity Questionnaire) ${ }^{12}$, smoking and alcohol consumption ${ }^{13}$. Nutrition knowledge was assessed with the General Nutrition Knowledge Questionnaire ${ }^{14}$. Job satisfaction was measured with an adapted version of a validated job satisfaction questionnaire ${ }^{15,16}$. Furthermore, an evaluation section was added at follow-up based on the intervention components. The questionnaire took approximately 30 minutes to complete.

Spot Urine Sample

For assessment of objective biomarkers of diet, a non-fasting urine sample $(20 \mathrm{ml})$ was collected at each time point. Urine samples were collected on the same day as the ON-duty 24h-diet recall so that Vitamin C intake ON duty could be assessed ${ }^{17}$. The Vuilleumier and Keck method was followed to analyze the urine samples ${ }^{18}$.

Interviews 
Semi-structured interviews were carried out with employees at baseline and follow-up assessing their views on healthy eating and the intervention. The aim was to interview between 10 and 20 employees who represented different age groups and gender until data was saturated. Managers $(n=4)$ who were involved in organizing the intervention were also interviewed. Questions were adapted from previous qualitative studies ${ }^{19,20}$. Each interview took approximately 20 minutes. Baseline interviews were conducted by the researcher who managed the intervention (Supplementary Table 1). The interview questions postintervention were adapted to also assess employee's views on the intervention. These were conducted by a second researcher who was not involved in delivering the intervention to increase the likelihood of unbiased feedback.

\section{Intervention}

The intervention was informed by the literature and in discussion with management and staff. Nutrition intervention components were based on the Eatwell Plate ${ }^{21}$ and were carried out by one of the researchers, as described in Table 1, who is a qualified nutritionist. Table 1 also specifies all behavior change techniques applied according to the CALO-RE Taxonomy ${ }^{22}$. The main components were a healthy eating policy, which was introduced by the company, free fruit for staff, diet group education and one individual diet and health consultation with the research nutritionist. 


\section{Analysis}

Quantitative analysis

Collected data from the questionnaires and health assessments was analyzed with SPSS vs 20. Descriptive statistics at baseline were reported as mean (SD) for continuous data and frequencies (percentages) for categorical data. Analysis of pre- and post-intervention data within the different genders was analyzed with paired samples t-test for continuous variables and Pearson's Chi-square for categorical variables. Statistical significance was indicated as $\mathrm{p} \leq 0.05$. Independent samples t-tests were utilised to examine differences between genders.

\section{Nutrient Intake Analysis}

The dietary analysis software package WISP (Tinuviel Software, Warrington, UK) was used for entering all diet recalls ${ }^{23}$. Dietary information was then extracted into SPSS for analysis of the nutrients of interest (total energy (TE), energy from fat, saturated fat (SFA), sugar (NMES), fruit and vegetables (FV), sodium, fiber, Vitamin C). To reduce inter-observer error, baseline and follow-up recalls were entered by one researcher and checked for accuracy by a second researcher.

\section{Qualitative analysis}

All interviews were transcribed into Microsoft Word and identifiable information was removed from the typed transcripts. The original records were destroyed and the transcript analyzed using thematic analysis ${ }^{24}$. Codes and candidate themes were identified and grouped into main themes. 


\section{Results}

At baseline, 79 out of 83 employees were eligible and agreed to participate in the study. The mean age was 27.7 years for males (65\%) and 31.1 years for women. Non-managerial positions were held by $78 \%$ of men and $70 \%$ of women.

\section{Compliance}

All participants completed all baseline assessments and 60 participants completed the six-month follow-up assessment. Participants who did not complete the follow-up visits had left the company (n=14), were on long-term sick leave $(n=1)$, or followed a strict weight gain or loss diet $(n=3)$. During the intervention period, the company encouraged employees to attend all study-related activities during working hours. Compliance for each intervention component is listed in Table 1.

\section{Health measures}

Baseline and change statistics for health measures are demonstrated in Table 2. The mean BMI for both genders was in the overweight category. At baseline, men were in the pre-hypertensive category, whereas females on average had a healthy blood pressure. At follow-up there was no change in weight and BMI, however, females appeared to have their $\mathrm{WC}$ reduced $(-2.2 \mathrm{~cm}, \mathrm{SD}: 4.9$, p-value $=0.06)$. There was a significant drop in SBP for both genders (males: $-3.3 \mathrm{mmHg}$, SD: 9.9, p-value $<0.05$ and females: -8.0 mmHg, SD: 7.7, p-value < 0.001) and in DBP for females only $(-3.6 \mathrm{mmHg}$, SD: 6.7, p-value < 0.05). With regards to lifestyle behaviors, at baseline and follow-up, males, on average, were more active, exceeded the recommended units of alcohol consumption per week ${ }^{25}$ more often, and were more likely to smoke 
compared to females. There was no significant change in physical activity behavior, smoking or alcohol consumption over the study period (results not shown).

Diet measures (baseline)

At baseline, TE intake was within the recommended energy allowance, except OFF duty, when males consumed more than the recommended 2500kcal/ day ${ }^{26}$ (2794.0 kcal, SD: 1277.5) (Table 3). Overall fat intake was higher than recommended for males and females OFF duty ${ }^{26}$ (37.9\% of TE, SD: 9.4 and 38.3, SD: 12.4 , respectively). Males and females consumed a similar amount of non-milk-extrinsic sugars (NMES) ON and OFF duty which was twice the amount recommended ${ }^{27}$ (ON duty, males: $17.2 \%$ of TE, SD: 17.3 ; females: $10.6 \%$ of TE, SD: 7.8 ). Males and females did not consume the recommended daily 5 portions of FV and on average consumed more fruit than vegetables. On average, males and females fell short of the $30 \mathrm{~g} /$ day recommendations for fiber ${ }^{27}$. Males far exceeded the recommendations for sodium intake ( $<2400 \mathrm{mg})$.

\section{Diet measures (follow-up)}

At follow-up, males significantly reduced TE consumption OFF duty (-449.5 kcal, SD: 1035.1, p-value $\leq$ $0.01)$ and significantly dropped their sugar intake ON duty ( $8.7 \%$ of TE, SD: $20.1 ; p$-value: $\leq 0.01)$. Females also reduced their NMES intake ON duty, which was not statistically significant (3.9\%, SD: 9.1; p-value: 0.062), and there was no improvement OFF duty. Total fat, SFA, fiber and sodium intake did not change. Females significantly increased their vegetable intake OFF duty (93.9 g, SD: 194.6, p-value $\leq 0.05)$ and there was no significant change in FV consumption in males. In contrast to the 24-hour diet recall, the results from the diet-related questions in the questionnaire suggest that consumption of fruit servings did 
significantly increase (from 2.4 portions, SD: 1.3 to 2.8 portions, SD: 1.3 ; p-value $<0.05$ ) and that the number of days that participants reported to consume fruit also increase by almost one day (from 4.5 days, SD: 2.0 to 5.4 days, SD: 1.8 ; p-value $<0.001$ ). The questionnaire did not find a change in vegetable or takeaway consumption. There was no significant change in dietary or urinary Vitamin C.

\section{Nutrition knowledge}

Nutrition knowledge increased by 2.2 points in males (SD: 8.8 , p-value: 0.132 ), although this was not statistically significant, and significantly increased in females by 5.6 points (SD: 8.3 , p-value: $\leq 0.01$ ) over the intervention period (Supplementary Table 2).

\section{Work-related outcomes}

The company reported a drop in sickness absenteeism by 40 days and employee retention rate increased by $20 \%$ post-intervention compared to the previous year. There was a change in job satisfaction as can be seen in Supplementary Table 3. On average, participants felt significantly less supported at work (before: 7.5 SD: 1.7; after: 7.0 SD: 1.8; p-value < 0.05). Although there was a slight increase in feeling that the employer cared about staff's wellbeing, this was not statistically significant.

\section{Intervention evaluation}

Participant feedback collected after the intervention period suggests that the intervention was well received by the participants and $91 \%$ felt that it had been beneficial for the company. In particular, more than $80 \%$ of staff felt that the presentations, the healthy eating policy, the free fruit and the meetings with the 
researcher helped them to make changes to their diet (Table 4). The goal setting sheets and the rewards were scored mostly neutral.

Interviews (baseline)

The interviews were conducted with 17 participants ( 9 males, 8 females) and four managers ( 2 males, 2 females), including the director.

\section{a) Employees}

There were four main themes identified that were discussed by employees when asked about their current diet knowledge, food choice influences and expectations on the study which were: (I) social context, (II) individual factors, (III) work, (IV) education. Table 5 provides an overview of detected sub-themes, codes and selected quotes that best describe each code.

\section{b) Managers}

Interviews with the managers strongly suggested that the manager's key priority in introducing this intervention was to improve staff's health long-term.

'It's talking about long-term health; it's not just about the next six months. It's trying to teach them better habits for life. [...] it also makes you stand out as an employer [...] we're trying to retain staff [...] offer them better benefits as part of their role'’ Manager, Female 
Managers fully supported the researchers in the recruitment and intervention process and anticipated that staff would view this intervention as beneficial and that the business would stand out for its corporate responsibility.

'So [the vending machines are] going to go. The idea of fruit about the place - good. The idea of bringing food in, hot food that's gonna be the right balance - great. I just like the whole idea. So now I'm a hundred percent on your side, things are going to change.' Manager, Male

With regards to concerns about the success of the intervention, managers agreed that staff had to buy into the healthy eating concept and provide support amongst one another. Furthermore, regular activities and contact with the researcher were felt to be important to maintain staff's interest and participation.

Interviews (follow-up)

\section{a) Employees}

Twelve employees ( 7 males, 5 females) were interviewed as well as the same four managers. Four participants who were interviewed pre-intervention were included in the follow-up interviews. Eight participants who were included in the pre-intervention interviews had either left the company or did not want to be interviewed again. A number of key themes identified in the follow-up interviews were identical to baseline findings. Table 6 mainly lists newly identified key- and sub-themes identified: (I) workenvironment, (II) social context, (III) self-improvement, (IV) intervention. In comparison to preintervention interviews, participants more often reported awareness of food groups, consumption of wholegrains, nutrient deficiencies and the effects that diet can have on energy levels. Individual factors, e.g. convenience and cost were still important, however, it was now more convenient to eat healthy than unhealthy due to the healthy eating policy and the free fruit. Interviewees felt the policy affected 
participants' eating habits at home as well as in work. Participants most often reported to have stopped fizzy drink consumption and to choose healthier snack alternatives at work. Social context was still important in influencing food choice; however, some interviewees reported to provide healthier food at home post-intervention and therefore have become a positive influence on their family. When asked about what helped employees to change their diet and what they enjoyed most, four points were prominent: (I) the healthy environment (i.e. healthy eating policy and access to fruit), (II) the management lead by example and strongly supported the intervention, (III) the team effort and support that came from colleagues and (IV) the knowledge and expertise from the researcher who led the study. The policy that was introduced by the management was first perceived with surprise and some reservations but at follow-up, it was perceived that this has helped people to change their diet.

\section{b) Managers}

All managers spoke positively about the intervention and agreed that their expectations have been met.

'[...] my expectations were very high, given where our staff were at and what they were consuming on a daily basis [...] we were very concerned for our staff and their well-being, [...] you want to get the most out of your workforce and make sure they are here, day in and day out. [...] Ah, without a shadow, without a doubt - yes! Yes, it's been fantastic, couldn't speak highly enough of it.' Manager, Male

Managers felt that the study wouldn't have been as successful if staff would have been allowed to eat unhealthy foods in work and that the supply of the free fruit was a worthwhile investment. In line with the staff feedback, management reported that they are going to continue with the changes that have been introduced. 


\section{'[...] it's now embedded in the company culture that you don't bring fast food or takeaway into the premises} and that is going to continue on.' Manager, Female

The interviews demonstrated that management was engaged with the intervention, participated in the activities and made positive changes to their diet themselves. Managers reported that working with the researchers on the delivery of the intervention was important to obtain staff buy-in. The interviewed managers also reported that good communication of the changes as well as the buy-in and participation of all managers was key to intervention success.

\section{Discussion}

\section{Findings}

To our knowledge, this was one of the first nutrition interventions that incorporated a strict food policy in a workplace setting. Previous studies, such as the UCLA WORKING study, are examples where so-called 'push' strategies (e.g. plan for exercise breaks during meetings) were used, rather than 'pull' (e.g. offer free gym passes) strategies, which is similar in its approach to this study in that healthy choices were made the easy or default choice and unhealthy choices were more difficult to carry out ${ }^{28}$. While the food policy was controversial at the start, staff adapted to it quickly and the findings from the evaluation questionnaire and qualitative interviews suggest that the majority appreciated the changes and that the company is going to keep the changes post-intervention. Compared to findings from previous interventions where participation rates were reported around a median of $33 \%{ }^{29}$, this pilot study had a very high level of participation and compliance with study components, which is likely due to the high management buy-in and time given at work for participation. 
Looking at the change in eating behavior ON and OFF duty; participants significantly reduced their NMES intake during working days only, which suggests that the food policy was effective in limiting the consumption of foods and drinks high in sugar at work. At follow-up, females and males NMES consumption was closer to, but still not in line with the recommendations (5.7\% of TE and $9.7 \%$ of TE, respectively). This was confirmed in the follow-up interviews, as a number of people reported to have stopped drinking sugary drinks at work. Despite the fact that fast food was not allowed to be consumed within the company, there was no change in total or SFA intake. The interviews suggest that taste is important when choosing food. This may be the reason why there was no change in fat or SFA intake in study participants. Fat adds flavour to food and, if study participants reduced their sugar intake, a reduction in fat intake would likely have resulted in lack of flavour.

The results from the 24-hour diet recalls and the urinary analysis did not show a significant increase in fruit consumption during working days, despite the free fruit available at work, and the favourable comments regarding the fruit provision during interviews. This is in line with the lack of increase in reported FV intake. The relatively small increase in FV consumption is similar to findings from other workplace interventions ${ }^{2,30}$ and the lack of statistical significance in this study may have been due to the limited number of participants. The benefit for free fruit provision has been supported by a recent quasiexperimental study where workplaces either provided fruit free of charge, charged $\$ 0.50$ or $\$ 1$. Employees in the workplaces where fruit was provided free of charge consumed significantly more fruit compared to the other workplaces ${ }^{31}$. Nutrition knowledge only increased significantly in females, suggesting that females learned more during the intervention. As a higher nutrition knowledge score is associated with better diet quality ${ }^{32}$, women may be more likely to eat a healthier diet. This, however, was not reflected in the diet change outcomes of this study. Qualitative feedback also suggested that people's diet pre-and postintervention was strongly influenced by the social context at home and therefore incorporating relatives and friends in an intervention may be important. Sorensen et al. were one of the few who found that the 
workplace + family intervention was more effective in increasing FV intake than the intervention only focusing on staff ${ }^{33}$. The difference in eating habits ON and OFF duty at baseline and follow-up, as well as the importance of social influence on eating suggests that future interventions should aim to target eating habits at home and at work.

Two of the main findings from the qualitative and quantitative evaluation were that healthy eating had become the most convenient choice at work due to the ban on junk food and the provision of fruit and that management was supportive of the intervention. This is in line with the findings from other studies that support the inclusion of environmental strategies and to secure management buy-in ${ }^{34}$ to establish a culture of health ${ }^{35}$. Interviewees also mentioned that different parts of the intervention helped them to improve their diet, suggesting that a multi-component approach works well in the workplace setting which agrees with findings from other authors ${ }^{36,37}$.

With regards to health measures, SBP changed in both genders and DBP in females only. There was no significant change in weight, BMI or WC, despite the ban of unhealthy foods at work. This is in line with the main aims of this intervention, since the company did not want to focus on weight loss but on improving overall diet and health. Other interventions similarly reported no change or only a small change in body weight whereas others have reported weight loss up to $8.8 \mathrm{~kg}{ }^{38}$. The lack of overall weight loss could also be explained by the variety of goals that study participants set out at the start (e.g. increase FV or water intake; reduce snack, or alcohol consumption; weight loss). The goal setting component has not been analyzed further here and is an area worthwhile investigating in future as it has been found an effective weight loss strategy ${ }^{39}$.

Change in work-related outcomes reported here may not be solely accredited to the diet intervention but may partially result from the improvement in health and diet. Evidence from previous diet interventions on business benefits is limited, which is likely due to the lack of a standardised measurement of company 
benefits and possibly lack of access to company records. The findings suggest staff felt significantly less supported at work, despite the diet support. Although not significant, the small increase in the feeling that the employer cared about their well-being, suggests there were other work-related factors that were not assessed here which made employees feel less supported overall.

\section{Strengths and limitations}

Intervention strengths include the high participation and attendance rate, the rich data set collected, including information on the difference in eating habits ON and OFF duty, the urinary biomarkers and the mixed-methods evaluation. Although these results do not lead to conclusions on the long-term effectiveness of the intervention beyond the six months, it has been suggested that this period is long enough for any immediate benefits, that may not last, to wear off ${ }^{40}$.

A limitation of most dietary research is the reliance on self-reported data and the potential for underreporting. Particularly female participants reported a very low kcal intake at baseline. Social desirability bias may have influenced dietary findings and ideally, a second researcher would have collected all outcome data. However, this was not feasible for this study. Furthermore, the use of urinary ascorbic acid as a biomarker can be insensitive in picking up small changes in Vitamin C intake and doubly labelled water might be more informative to measure accuracy of self-reported diet intake ${ }^{41}$. The lack of a control group allows us to make only limited conclusions concerning the outcomes. This is a common issue as employers seem most interested in pre- and post-interventions to receive best possible outcomes for their staff and business, and would be unwilling participate in a RCT where allocation to a control group is an option ${ }^{42,43}$. In addition, due to the inclusion of several questions measuring similar outcomes there is an increased likelihood of type I error. In multi-component interventions it is common that not all intervention 
components work out as planned ${ }^{44}$. This is why a process evaluation ${ }^{2,43}$ and keeping the intervention flexible is important.

\section{Recommendations}

Findings from this pilot study will be valuable to provide variability data on the primary endpoint on which to base a formal power calculation for a larger, randomised study in future. Key learning points for future interventions include:

- nudging the environment to make healthy choices more, and unhealthy choices less accessible

- securing management buy-in and support throughout the intervention

- using a multi-component approach that allows participants to engage in different diet-related activities

- having a flexible approach and tailoring the intervention, if required

- stressing the importance of healthy eating habits at home and at work to effect overall diet change

- working with professionals

- Including staff in decision making and delivery of the intervention for a low-cost, long-term solution.

\section{SO WHAT?}

\section{What is already known on this topic?}

Increasing healthy food options in work canteens is a commonly used strategy in workplace dietary interventions. Findings from those studies have reported a small increase in fruit and vegetable intake and sometimes small reductions in weight loss. 


\section{What does this article add?}

Findings from this study suggest that banning unhealthy foods from the company as part of a multicomponent workplace intervention can significantly reduce sugar intake in males and improve systolic blood pressure in young employees working at medium-sized businesses. Strong management support seems necessary for such a success.

\section{What are the implications for health promotion practice or research?}

Nudging the work environment to make unhealthy choices less and healthy choices more available should be further explored in dietary workplace research as it may lead to behavior change. Researchers and policymakers need to engage management to positively drive such change. 


\section{References}

1. Ni Mhurchu C, Aston LM, Jebb SA. Effects of worksite health promotion interventions on employee diets: a systematic review. BMC Public Health. 2010;10:62.

2. Geaney F, Kelly C, Greiner BA, Harrington JM, Perry IJ, Beirne P. The effectiveness of workplace dietary modification interventions: A systematic review. Prev Med. 2013;57(5):438447.

3. Quintiliani L, Poulsen S, Sorensen G. Healthy eating strategies in the workplace. Int J Work Health Manag. 2010;3(3):182-196.

4. Deliens T, Clarys P, De Bourdeaudhuij I, Deforche B. Determinants of eating behaviour in university students : a qualitative study using focus group discussions. Br Med J. 2014;14(53):112.

5. Pelletier JE, Graham DJ, Laska MN. Social norms and dietary behaviors among young adults. Am J Health Behav. 2014;38(1):144-152.

6. Han E, Powell LM. Consumption Patterns of Sugar-Sweetened Beverages in the United States. $J$ Acad Nutr Diet. 2013;113(1):43-53.

7. Allman-Farinelli M, Partridge SR, Roy R. Weight-Related Dietary Behaviors in Young Adults. Curr Obes Rep. 2016;5:23-29.

8. Lachat C, Nago E, Verstraeten R, Roberfroid D, Van Camp J, Kolsteren P. Eating out of home and its association with dietary intake : a systematic review of the evidence. Obes Rev. 2012;13(April):329-346.

9. Deliens T, Van Crombruggen R, Verbruggen S, De Bourdeaudhuij I, Deforche B, Clarys P. 
Dietary interventions among university students: A systematic review. Appetite. 2016;105:14-26.

10. Craig P, Dieppe P, Macintyre S, Michie S, Nazareth I, Petticrew M. Developing and evaluating complex interventions: the new Medical Research Council guidance. Int J Nurs Stud. 2013;50(5):587-92.

11. Geaney F, Scotto di Marazzo J, Kelly C, et al. The food choice at work study : effectiveness of complex workplace dietary interventions on dietary behaviours and diet-related disease risk study protocol for a clustered controlled trial. Trials. 2013;14:370.

12. Craig CL, Marshall AL, Sjöström M, Bauman AE, Booth ML, Ainsworth BE, Pratt, M, Ekelund U, Yngve A, Sallis JF, Oja P. International physical activity questionnaire: 12-country reliability and validity. Med Sci Sports Exerc. 2003;35(8):1381-1395.

13. Harrington, J., Perry, I., Lutomski, J., et al. (2008) SLÁN 2007: Survey of Lifestyle, Attitudes and Nutrition in Ireland. Dietary Habits of the Irish Population, Department of Health and Children. Dublin: The Stationery Office.

14. Parmenter K, Wardle J. Development of a general nutrition knowledge questionnaire for adults. Eur J Clin Nutr. 1999;53(4):298-308.

15. Judge TA, Locke EA, Durham CC, Kluger AN. Dispositional Effects on Job and Life Satisfaction : The Role of Core Evaluations. J Appl Psychol. 1998;83(1):17-34.

16. Thøgersen-Ntoumani C, Fox KR, Ntoumanis N. Relationships between exercise and three components of mental well-being in corporate employees. Psychol Sport Exerc. 2005;6(6):609627.

17. McGrath A, Hamill L, Graham G, et al. Urinary vitamin C excretion as a biomarker of 
compliance in a fruit and vegetable intervention study. Proc Nutr Soc. 2013;72:E154.

18. Vuilleumier JP, Keckk E. Fluorometric assay of vitamin C in biological materials using a centrifugal analyser with fluorescence attachment. J Micronutr Anal. 1989;5:25-34.

19. Pridgeon A, Whitehead K. A qualitative study to investigate the drivers and barriers to healthy eating in two public sector workplaces. J Hum Nutr Diet. 2013;26(1):85-95.

20. Fitzgerald S, Geaney F, Kelly C, Mchugh S, Perry IJ. Barriers to and facilitators of implementing complex workplace dietary interventions : process evaluation results of a cluster controlled trial. BMC Health Serv Res. 2016;16:139.

21. Eatwell plate. Food Standards Agency. http://webarchive.nationalarchives.gov.uk/+http://www.food.gov.uk/northernireland/nutritionni/niyoungpeople/survivorform/breadandbutterstuff/eatwellplate. Published 2007. Accessed August 8, 2016.

22. Michie S, Ashford S, Sniehotta FF, Dombrowski SU, Bishop A, French DP. A refined taxonomy of behaviour change techniques to help people change their physical activity and healthy eating behaviours: The CALO-RE taxonomy. Psychol Health. 2011;26(11):1479-1498.

23. Chan W, Brown J, Lee S. Meat, Poultry and Game. Fifth Supplement of McCance \& Widdowson's. The Composition of Foods. 5th ed. Cambridge and London: The Royal Society of Chemistry and the Ministry of Agriculture Fisheries and Food; 1995.

24. Braun V, Clarke V. Using thematic analysis in psychology. Qual Res Psychol. 2006;3:77-101.

25. Department of Health. UK Chief Medical Officers' Alcohol Guidelines Review: Summary of the Proposed New Guidelines. 2015. 
26. Department of Health. Dietary Reference Values of Food Energy and Nutrients for the United Kingdom: Report of the Panel on Dietary Reference Values of the Commitee on Medical Aspects of Food Policy. Great Britain; 1991.

27. Scientific Advisory Committee on Nutrition. Carbohydrates and Health. London: TSO; 2015.

28. Hopkins JM, Glenn BA, Cole BL, McCarthy W, Yancey A. Implementing organizational physical activity and healthy eating strategies on paid time: process evaluation of the UCLA WORKING pilot study. Health Educ Res. 2012;27(3):385-398.

29. Robroek SJW, Lenthe FJ Van, Empelen P Van, Burdorf A. Determinants of participation in worksite health promotion programmes : a systematic review. Int J Behav Nutr Phys Act. 2009;6:26.

30. Pomerleau J, Lock K, Knai C, McKee M. Interventions designed to increase adult fruit and vegetable intake can be effective: a systematic review of the literature. J Nutr. 2005;135(10):2486-2495.

31. Pescud M, Waterworth P, Shilton T, et al. A healthier workplace? Implementation of fruit boxes in the workplace. Health Educ J. 2016;1(12).

32. Geaney F, Fitzgerald S, Harrington JM, Kelly C, Greiner BA, Perry IJ. Nutrition knowledge, diet quality and hypertension in a working population. Prev Med Reports. 2016;75(7).

33. Sorensen G, Stoddard A, Peterson K, et al. Increasing fruit and vegetable consumption through worksites and families in the treatwell 5-a-day study. Am J Public Health. 1999;89(1):54-60.

34. Goetzel RZ, Roemer EC, Short ME, et al. Health improvement from a worksite health promotion private-public partnership. J Occup Environ Med. 2009;51(3):296-304. 
35. Goetzel RZ, Henke RM, Tabrizi M, et al. Do Workplace Health Promotion ( Wellness ) Programs Work? J Occup Environ Med. 2015;57(1):e10.

36. Maes L, Van Cauwenberghe E, Van Lippevelde W, et al. Effectiveness of workplace interventions in Europe promoting healthy eating : a systematic review. 2012;22(5):677-683.

37. Kahn-Marshall JL, Gallant MP. Making Healthy Behaviors the Easy Choice for Employees: A Review of the Literature on Environmental and Policy Changes in Worksite Health Promotion. Heal Educ Behav. 2012;39(6):752-776.

38. Weerasekara YK, Roberts SB, Kahn MA, LaVertu AE, Hoffman B, Das SK. Effectiveness of Workplace Weight Managment Interventions: a Systematic Review. Curr Obes Rep. 2016;5(2):298-306.

39. Pearson ES. Goal setting as a health behavior change strategy in overweight and obese adults: A systematic literature review examining intervention components. Patient Educ Couns. 2012;87(1):32-42.

40. Janer G, Sala M, Kogevinas M. Health promotion trials at worksites and risk factors for cancer. Scand J Work Environ Health. 2002;28(3):141-157.

41. Hill RJ, Davies PSW. The validity of self-reported energy intake as determined using the doubly labelled water technique. Br J Nutr. 2001;85(4):415-430.

42. Pelletier K. A review and analysis of the clinical and cost-effectiveness studies of comprehensive healthl promotion and disease management programs at the worksite: 1995-1998 ubdate (IV). Am J Health Promot. 1999;13(6):333-345.

43. O'Donnell MP. What is the ROI for Workplace Health Promotion? It Really Does Depend, and 
That's the Point. Am J Health Promot. 2015;29(3):v-viii.

44. Montano D, Hoven H, Siegrist J. Effects of organisational-level interventions at work on employees ' health : a systematic review. BMC Public Health. 2014;14:135. 
Tables:

Table 1. Description of implemented intervention components

Table 2. Change in health measures in males and females

Table 3. Change in ON and OFF duty eating habits in males and females

Table 4. Evaluation questionnaire post-intervention $(n=60)$

Table 5. Thematic evaluation of employee interviews (pre-intervention)

Table 6. Thematic evaluation of employee interviews (post-intervention)

Figures:

Figure 1: Study design of the six-month multicomponent diet intervention

Figure 2: Outline of study appointments carried out at baseline and follow-up

Supplementary Material:

Supplementary Table 1. Employee interview guide (pre-intervention)

Supplementary Table 2. Nutrition knowledge in males and females pre- and post-intervention

Supplementary Table 3. Job satisfaction pre- and post-intervention 